\title{
Centrifuge model tests on shallow overburden tunnels with pre- ground improvement to clarify seismic behavior
}

\section{$\operatorname{AUTHOR}(S):$}

Sawamura, Y.; Konishi, K.; Kishida, K.; Kimura, M.

\section{CITATION:}

Sawamura, Y.... [et al]. Centrifuge model tests on shallow overburden tunnels with pre-ground improvement to clarify seismic behavior. Tunnels and Underground Cities. Engineering and Innovation Meet Archaeology, Architecture and Art: Proceedings of the WTC 2019 ITA-AITES World Tunnel Congress (WTC 2019), May 3-9, 2019, Naples, Italy 2019: 1528-1537

\section{ISSUE DATE:}

2019-04-17

URL:

http://hdl.handle.net/2433/241265

\section{RIGHT:}

This is an Accepted Manuscript of a book chapter published by CRC Press in Tunnels and Underground Cities. Engineering and Innovation Meet Archaeology, Architecture and Art: Proceedings of the WTC 2019 ITA-AlTES World Tunnel Congress (WTC 2019), May 3-9, 2019, Naples, Italy on 17 April 2019, available online: http://www.crcpress.com/9780429424441.; The full-text file will be made open to the public on 17 April 2020 in accordance with publisher's 'Terms and Conditions for Self-Archiving'.; この論文は出版社版でありません。引用の際に は出版社版をご確認ご利用ください。;This is not the published version. Please cite only the published version. 


\title{
Centrifuge model tests on shallow overburden tunnels with pre-ground improvement to clarify seismic behavior
}

\author{
Y. Sawamura, K. Konishi, K. Kishida \& M. Kimura \\ Kyoto University, Kyoto, Japan
}

\begin{abstract}
Due to the development of auxiliary construction methods, such as pre-ground improvement, the NATM is often used to excavate tunnels with a shallow overburden in a soft ground. The improvement stabilizes the tunnel face and suppresses the subsidence of the ground surface. In previous researches, although the optimum ground improvement area was examined through experiments and numerical simulations, the seismic behavior of the tunnels was not clearly discussed. In this study, dynamic centrifugal model experiments are conducted to clarify the dynamic behavior of shallow tunnels with pre-ground improvement. The experimental results indicate that when the entire ground around a tunnel is improved, the shear deformation of the tunnel can be suppressed. On the other hand, when the ground around the tunnel crown and top section is improved, the response of the tunnel is amplified by the concentration of weight at the upper part of the tunnel.
\end{abstract}

\section{INTRODUCTION}

Several problems arise when excavating a tunnel with a shallow overburden in a soft ground, such as the instability of the tunnel face and surface subsidence. In particular, because the loosening of the ground induced by the tunnel excavation expands to the surrounding ground, the influence will directly reach the ground surface. Traditionally, the cut and cover tunneling method has been widely used for the excavation of shallow overburden tunnels in soft grounds. Due to the technical development of auxiliary construction methods, however, the New Austrian Tunneling Method (NATM) is now often adopted for these shallow overburden tunnel excavations. Although the NATM is more economical than the cut and cover tunneling method or shield tunneling method, the NATM presupposes the formation of a ground arch in the surrounding ground and the stability of the tunnel face. For this reason, when a shallow overburden tunnel is to be constructed by the NATM, safe and appropriate tunnel face stabilization methods are required for the excavation.

In the constructions of Tohoku Shinkansen (Bullet Train) and Hokuriku Shinkansen, several tunnels with a shallow overburden were constructed in sandy soil mountains due to the linear constraints of the topography. Therefore, in order to secure the stability of the tunnel face and to suppress the subsidence of the ground, pre-ground improvement was applied before the tunnel excavations to sections with few restrictions on the ground segments. Figure 1 and Table 1 show the physical properties of the ground around the tunnels to which the pre-ground improvement method was applied. The area and the strength of the improved ground varied depending on the conditions, such as the overburden, the geological conditions, and the allowable settlement. The ground around the tunnel crown and top section was improved in Ushikagi Tunnel, while the entire ground around the tunnel was improved in Akahira Tunnel (Nonomura et al., 2013). Figure 2 shows the construction process of the pre-ground improvement method. Firstly, the ground is excavated from the ground surface to the crown of the tunnel, and cement is mixed with natural ground around the side wall of the tunnel using the shallow or deep mixing 


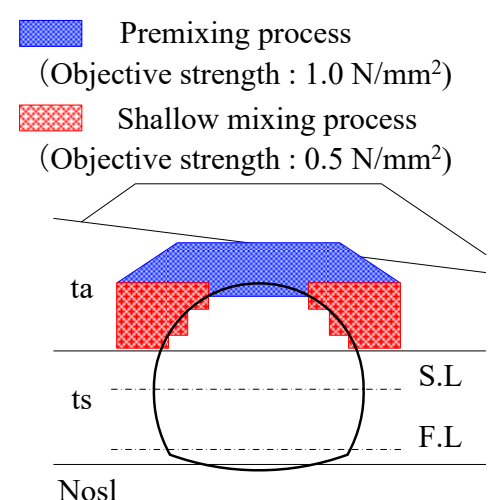

(a) Ushikagi Tunnel

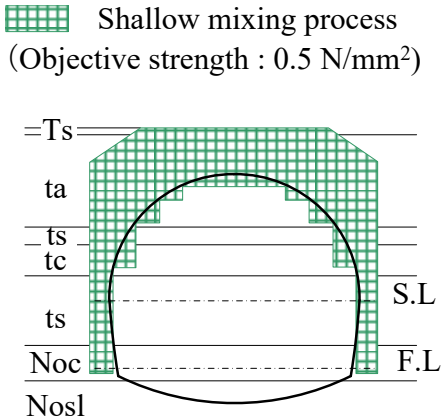

(b) Akahira Tunnel

Figure 1. Area and method of ground improvement around shallow overburden tunnel (After Nonomura et al., 2013).

Table 1. Physical properties of ground around tunnel with pre-ground improvement (After Nonomura et al., 2013)

\begin{tabular}{llllllll}
\hline & Symbol & $N$ value & $\gamma\left[\mathrm{kN} / \mathrm{m}^{3}\right]$ & $E\left[\mathrm{kN} / \mathrm{m}^{2}\right]$ & $v$ & $c\left[\mathrm{kN} / \mathrm{m}^{2}\right]$ & $\phi\left[^{\circ}\right]$ \\
\hline Ushikagi Tunnel & & & & & & & \\
Volcanic ash & ta & $2-4$ & 14 & $3.5 \times 10^{3}$ & 0.35 & 36 & 0 \\
Sandy soil & ts & $10-15$ & 18 & $2.0 \times 10^{4}$ & 0.35 & 20 & 30 \\
Shallow mixing soil & - & - & 19 & $5.0 \times 10^{4}$ & 0.35 & 144 & 30 \\
Premixing soil & - & - & 19 & $1.0 \times 10^{5}$ & 0.35 & 288 & 30 \\
\hline Akahira Tunnel & & & & & & & \\
Surface soil & Ts & - & 15 & $5.0 \times 10^{3}$ & 0.4 & 13 & - \\
Volcanic ash & ta & $3-8$ & 15 & $1.3 \times 10^{4}$ & 0.4 & 30 & - \\
Sandy soil & ts & 7 & 17 & $1.8 \times 10^{4}$ & 0.35 & - & 31 \\
Cohesive soil & tc & $3-4$ & 15 & $1.0 \times 10^{4}$ & 0.4 & 57 & - \\
Sandy soil & ts & $7-22$ & 18 & $3.0 \times 10^{4}$ & 0.35 & - & 31 \\
Cohesive soil & Noc & 5 & 17 & $1.3 \times 10^{4}$ & 0.4 & 31 & - \\
Sandy soil & Nosl & $16-50$ & 20 & $1.3 \times 10^{5}$ & 0.3 & - & 38 \\
Shallow mixing soil & - & - & 19 & $1.0 \times 10^{5}$ & 0.35 & 288 & 30 \\
\hline
\end{tabular}

stabilization method. Then, the excavated soil and cement are premixed and backfilled. Finally, the excavated soil is compacted by rolling it on the ground surface. After these steps, the tunnel is excavated using the NATM. For the sections of these two tunnels for which the pre-ground improvement method was adopted, the ground surface subsidence was reported to have been suppressed and the tunnels were constructed securely (Nonomura et al., 2013). However, it should be kept in mind that the area and the strength of the improved ground were determined based on empirical judgment.

In previous researches, the optimum ground improvement area was examined through experiments and numerical simulations performed during the excavation process. Kishida et al. (2016) conducted a series of three-dimensional trapdoor experiments and corresponding FE analyses to evaluate the effect of the pre-ground improvement method during a tunnel excavation. In addition, the enhancement of tunnel stability resulting from the application of the ground improvement method was discussed. Based on the results, they reported that the advantages of the pre-ground improvement method can be presented as three issues, namely, the effect of shear reinforcement, the effect of earth pressure redistribution, and the effect of ground reinforcement. These three issues were seen as becoming even more effective as the width and the height of the improved ground increased. Cui et al. (2018) conducted 2D elasto-plastic FE analyses that simulated the excavation process for a tunnel with pre-ground improvement. They confirmed that the pre-ground improvement method was able to effectively prevent the settlement of the ground and the tunnel when the ground was improved down to the tunnel feet. Furthermore, the effect of the pre-ground improvement method was seen to slightly increase with an increase in the strength of the improved ground. However, the seismic behavior of the tunnel 


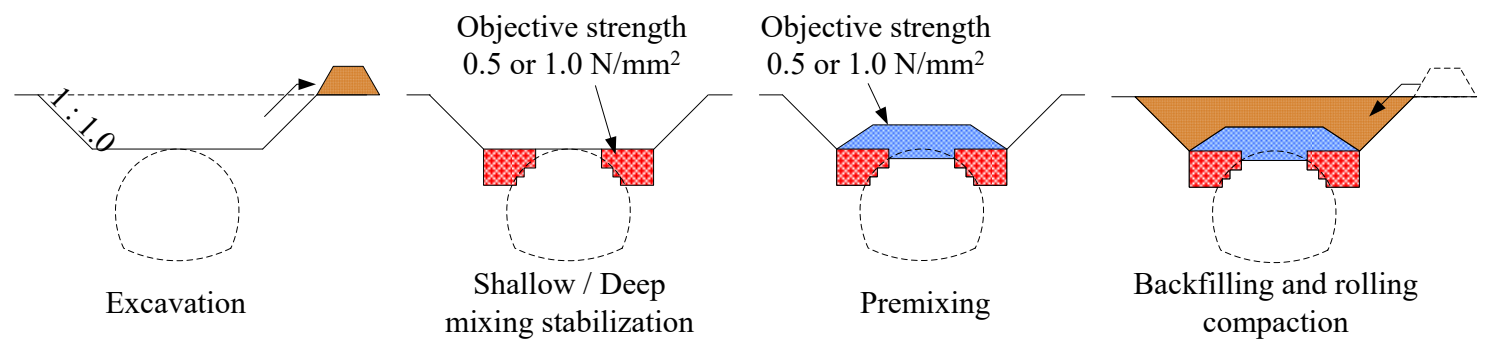

Figure 2. Construction process of pre-ground improvement method (After Kishida et al., 2016).

Table 2. Specifications of geotechnical centrifugal device at DPRI, Kyoto University

\begin{tabular}{ll}
\hline Specific & Geotechnical centrifuge \\
\hline Effective rotation radius [m] & 0.20 \\
Effective space for model installation $[\mathrm{m}]$ & $0.80(\mathrm{~L}) \times 0.36(\mathrm{~W}) \times 0.80(\mathrm{H})$ \\
Experiment capacity $[\mathrm{G} \times$ ton] & 24 \\
Maximum centrifuge acceleration $[\mathrm{G}]$ & 200 for static test, 50 for dynamic test \\
Maximum number of rotations $[\mathrm{rpm}]$ & 260 \\
\hline
\end{tabular}

with pre-ground improvement was not clearly discussed in their study. It is thought that the difference in stiffness between a soft ground and an improved ground strongly affects the stability of a tunnel during an earthquake.

In general, it is thought that mountain tunnels are seismic-resistant structures because they are surrounded by strong ground such as rock. However, within the current century, several mountain tunnels have suffered damage, for example, in the 2004 Mid-Niigata Prefecture Earthquake (Konagai et al., 2009; Jiang et al., 2010), the 2008 Wenchuan Earthquake (Wang et al., 2009; Shen et al., 2014), and the 2016 Kumamoto Earthquake (Zhang et al., 2018). Yashiro et al. (2007) analyzed the damage done to mountain tunnels in Japan due to earthquakes and classified this seismic damage into the following three categories: (1) Damage to shallow tunnels, (2) Damage to tunnels in poor geological conditions, and (3) Damage to tunnels due to fault slides. From this classification, it is confirmed that the seismic behavior of shallow overburden tunnels must be considered. The ground around these tunnels is often loose due to the small confining pressure; and thus, large shear deformation occurs during earthquakes. In such cases, it is highly possible that bending cracks will occur at the shoulder parts of the lining due to the bending moments generated in the lining (Yashiro et al., 2007).

Many researches have been conducted on the earthquake behavior of underground structures, such as tunnels, under the influence of ground shear deformation. Earthquake-resistant design methods for underground structures, including the free-field method, have been proposed (e.g., Wang, 1993; Hashash et al., 2001) On the other hand, as mentioned above, in terms of tunnels with pre-ground improvement, the effect of the improved ground on the seismic behavior of the tunnels has not been clarified. In this study, therefore, dynamic centrifugal model tests were conducted to clarify the dynamic behavior of shallow tunnels with pre-ground improvement.

\section{EXPERIMENTAL CONDITIONS}

\subsection{Experimental devices and objects}

In this study, dynamic shaking table tests were conducted under a gravitational acceleration of 50G using a geotechnical centrifugal device at the Disaster Prevention Research Institute (DPRI), Kyoto University. Table 2 shows the specifications of the geotechnical centrifugal device. The experimental subjects were two Shinkansen tunnels constructed by the NATM. The thickness of the overburden above the tunnels was set to $0.5 D$ ( $D$ : outer diameter of the tunnels) based on the construction records of NATM tunnels with pre-ground improvement. The improved ground patterns were the same as those adopted at actual construction sites, as shown in Figure 1 and Table 1. Figure 3 presents a schematic illustration of the experimental setup. A flexible shear beam soil container, $500 \mathrm{~mm}$ wide, $230 \mathrm{~mm}$ deep, and $400 \mathrm{~mm}$ high, was used. 


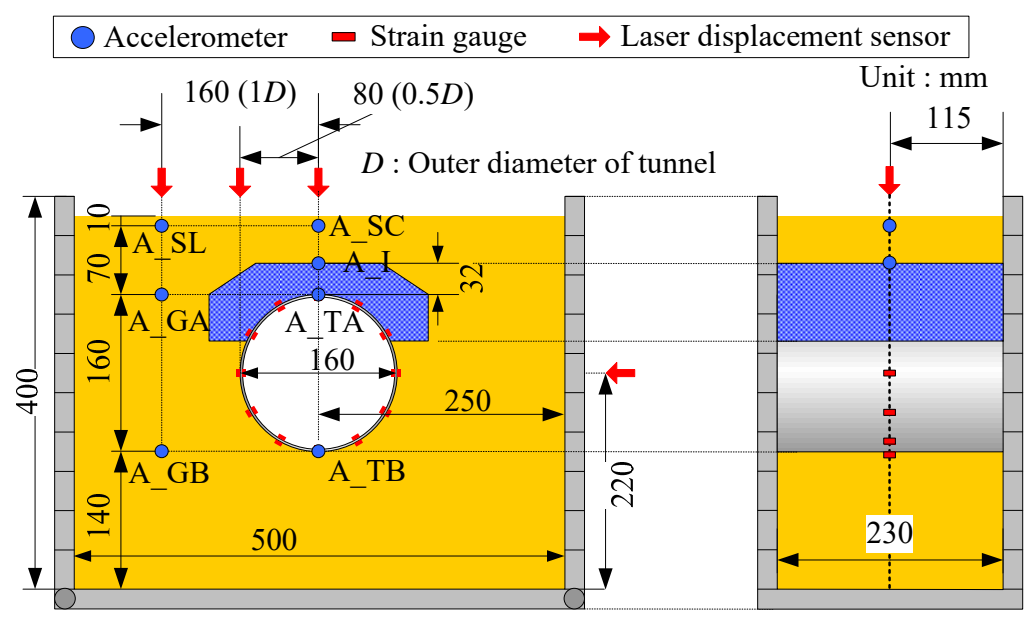

Figure 3. Schematic illustration of experimental setup (Case-2).

Table 3. Material properties of Toyoura sand

\begin{tabular}{ll}
\hline Specific gravity $G_{\mathrm{s}}$ & 2.64 \\
Average diameter $D_{50}[\mathrm{~mm}]$ & 0.20 \\
Internal friction angle $\phi[\mathrm{deg}]$ & 38.9 \\
Cohesion $c[\mathrm{kPa}]$ & 0 \\
Maximum void ratio $e_{\max }$ & 0.975 \\
Minimum void ratio $e_{\min }$ & 0.585 \\
Unit weight $\left(D_{\mathrm{r}}=85 \%\right) \gamma\left[\mathrm{kN} / \mathrm{m}^{3}\right]$ & 15.76 \\
\hline
\end{tabular}

The container consisted of 10 shear beams with a height of $40 \mathrm{~mm}$. Each shear beam could move independently during the shaking experiments. However, from the preliminary experiments using only soil samples, it was revealed that the reproducibility of the displacement of the side walls was poor. Therefore, the side walls were integrated with aluminum plates so that the soil container would permit the simple shear deformation of the model ground. Dry Toyoura sand was used as the ground material. Table 3 shows the physical properties of Toyoura sand. The model ground was constructed by compaction so that a relative density of $85 \%$ would be achieved.

\subsection{Experimental cases}

Figure 4 shows the three experimental cases. In Case-1, a tunnel without ground improvement was modeled despite a shallow overburden condition. Although this case would not be practically implemented, it is set for comparison with the two other cases with ground improvement. In Case-2, the ground around all the cross-sections of the tunnel was improved, while in Case-3, the ground around the crown of the tunnel and top section was improved. The improved ground patterns in Cases- 2 and -3 were determined by referring to actual construction records (Nonomura et al., 2013).

As a prerequisite for the experiments, rock bolts and shotcrete were not modeled because their effects on the seismic behavior of a tunnel are small and they are difficult to model. Moreover, the stress release of the ground around the tunnel during its excavation was not considered.

\subsection{Modeling of tunnels}

The model tunnels were manufactured so that their size and flexural rigidity would be as consistent as possible with the typical NATM tunnels of the Shinkansen sections, taking into consideration the boundary effect from the soil container. In addition, the model tunnels were manufactured as true circles because it is difficult to reproduce the shape of an actual tunnel. Consequently, the model tunnels were made of aluminum, as true circles, having an outer 


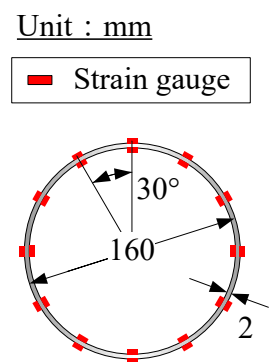

(a) Case-1

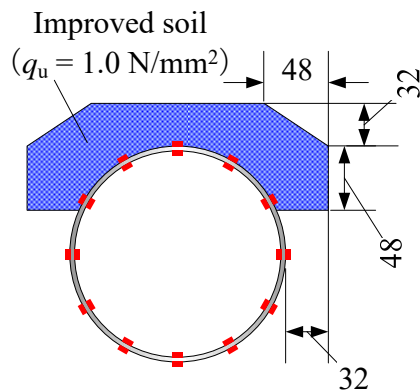

(b) Case-2

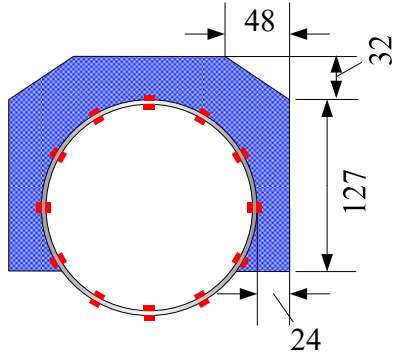

(c) Case-3

Figure 4. Experimental cases.

Table 4. Relationship between actual and model tunnels

\begin{tabular}{llll}
\hline & Actual tunnel $p$ & Model tunnel (prototype) $m_{\mathrm{p}}$ & Ratio $p / m_{\mathrm{p}}$ \\
\hline Young's modulus $E\left[\mathrm{~N} / \mathrm{mm}^{2}\right]$ & $2.20 \times 10^{7}$ & $7.06 \times 10^{7}$ & 0.31 \\
Outer diameter $D[\mathrm{~m}]$ & 10.1 & 8.0 & 1.26 \\
Thickness $t[\mathrm{~m}]$ & 0.30 & 0.10 & 3.0 \\
Axial stiffness $E A[\mathrm{kN}]$ & $2.03 \times 10^{8}$ & $1.75 \times 10^{8}$ & 1.16 \\
Bending stiffness $E I\left[\mathrm{kN} \cdot \mathrm{m}^{2}\right]$ & $2.44 \times 10^{9}$ & $1.37 \times 10^{9}$ & 1.78 \\
\hline
\end{tabular}

diameter of $160 \mathrm{~mm}$, a lining thickness of $2 \mathrm{~mm}$, and a depth of $225 \mathrm{~mm}$. Table 4 shows the relationship between an actual tunnel and a model tunnel.

\subsection{Modeling of ground improvement}

The pre-ground improvement was modeled with reference to the Japanese design manual for mountain tunnels. The improved ground was made by mixing Kasaoka clay $\left(w_{\mathrm{P}}=29.6 \%\right.$ and $w_{\mathrm{L}}$ $=62.1 \%$ ), high-early-strength cement, and water with the target of a uniaxial compressive strength $q_{\mathrm{u}}$ of $1.0 \mathrm{~N} / \mathrm{mm}^{2}$. The combined ratio of Kasaoka clay, high-early-strength cement, and water was $5: 1: 3.75$ by weight.

Originally, when the pre-ground improvement method was adapted for the in-situ construction of a tunnel excavation, as shown in Figure 2, the ground around the tunnel was improved prior to the tunnel excavation. In this experiment, however, in order to simplify the experimental conditions and to increase the quality of the improved ground, the improved ground was cast to the model tunnels beforehand and then each model tunnel with an improved ground was put into the soil container. Figure 5 shows the procedure for making the improved ground. The improved ground was removed from the mold form 48 hours after casting. The ground was cured in water for 5 days after casting and then dried in air for 48 hours.

In Cases-2 and -3, wherein the improved ground was attached to the tunnel model, in order to strengthen the adherence of the tunnel and the improved ground, several screw holes were made at the end of the tunnel (avoiding the area where the instruments were equipped), and screws were attached from the inside of the model tunnel (Figures 5 (a) and (d)). A 5.0-mm-thick sponge and a Teflon sheet were attached to both sides of the improved ground in the depth direction to reduce the friction between the improved ground and the soil container. Moreover, for the wall of the soil container, Teflon sheets were also attached parallel to the direction of excitation.

Table 5 shows the material constants of the improved ground. Compared with the in-situ construction, as shown in Figure 1 and Table 1, the density of the improved ground in this experiment was about the same as that of the surrounding ground, although the improved ground at an actual site is heavier than the surrounding ground. 


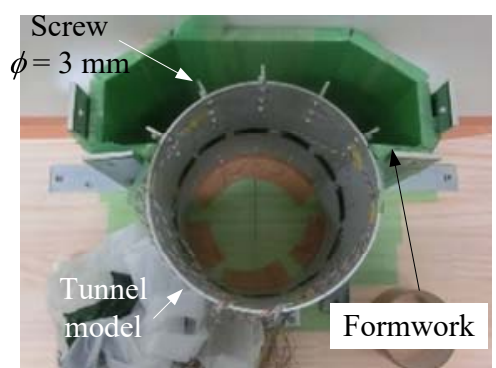

(a) Place formwork (Case-2)

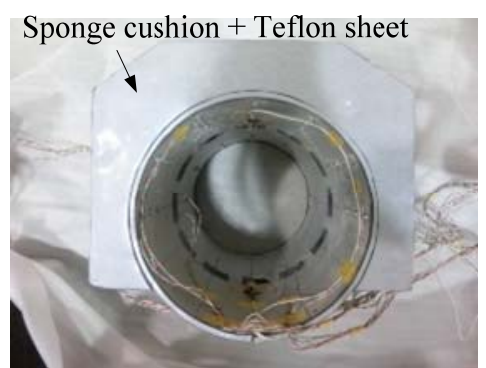

(c) Attach sponge cushion and Teflon sheet

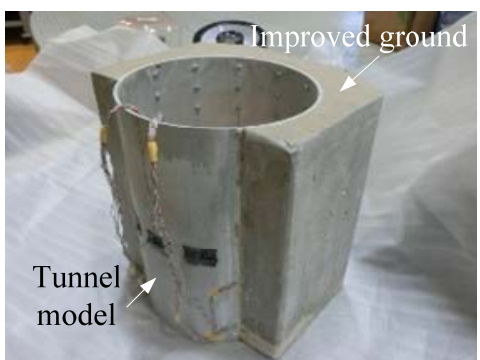

(b) Cast improved ground (Case-3)

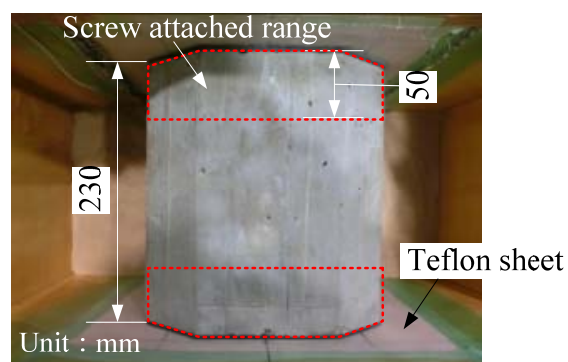

(d) Install into soil container

Figure 5. Casting-improved ground and installation into soil container.

Table 5. Material constants of improved ground

\begin{tabular}{ll}
\hline Young's modulus $E\left[\mathrm{kN} / \mathrm{m}^{2}\right]$ & $2.92 \times 10^{5}$ \\
Unit weight $\gamma\left[\mathrm{kN} / \mathrm{m}^{3}\right]$ & 15.67 \\
Compressive strength $f_{\mathrm{c}}\left[\mathrm{N} / \mathrm{mm}^{2}\right]$ & 1.01 \\
Tensile strength $f_{\mathrm{t}}\left[\mathrm{N} / \mathrm{mm}^{2}\right]$ & 0.29 \\
Poisson's ratio $v$ & 0.20 \\
\hline
\end{tabular}

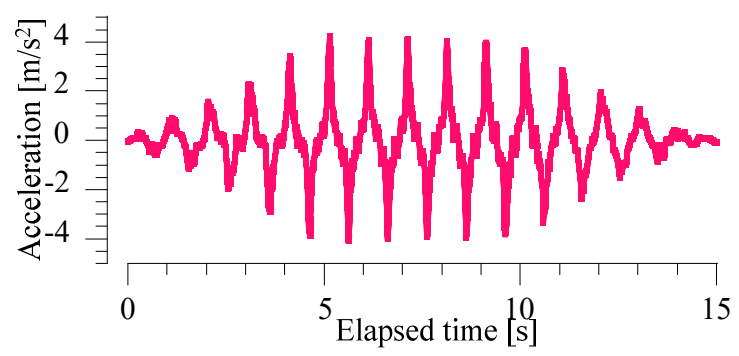

Figure 6. Time history of acceleration measured at shaking table (Case-1)

\subsection{Input waves and measurement items}

15 tapered sinusoidal waves, with a frequency of $1 \mathrm{~Hz}$ of the prototype, were input by controlling the displacement of the vibration table. The maximum acceleration of the input waves was about $4.0 \mathrm{~m} / \mathrm{s}^{2}$ in prototype scale. Figure 6 shows the time history of the input waves which were measured at the shaking table in Case-1. The response accelerations at the tunnel and the surrounding ground (right-hand side: positive), the strain occurring in the tunnel, and the horizontal displacement of the wall of the soil container (right-hand side: positive) were measured. 


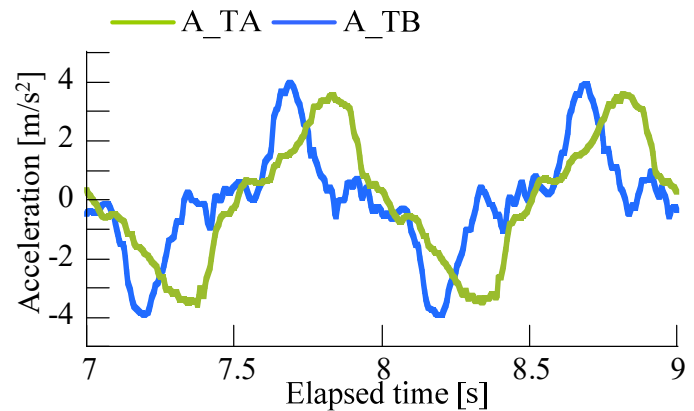

(a) Time histories of response accelerations (Case-1)

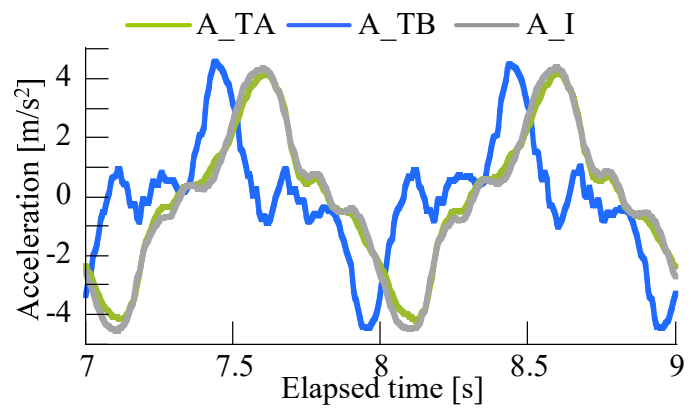

(c) Time histories of response accelerations (Case-2)

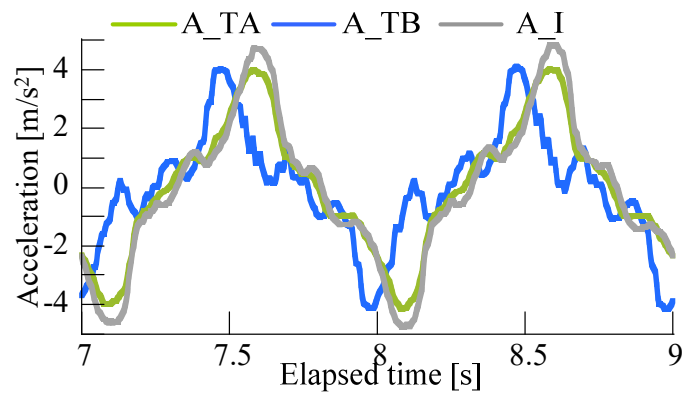

(e) Time histories of response accelerations (Case-3)

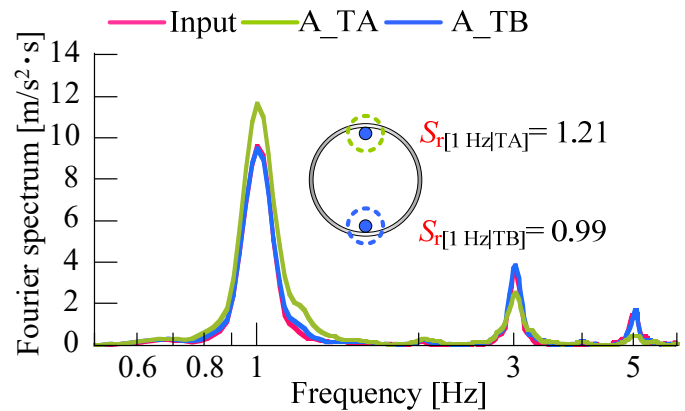

(b) Fourier spectrums (Case-1)

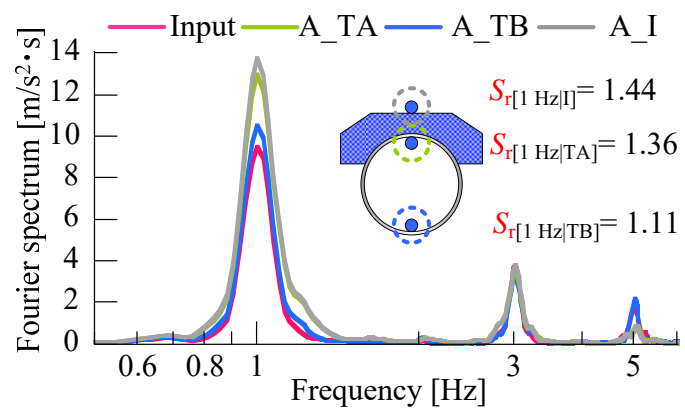

(d) Fourier spectrums (Case-1)

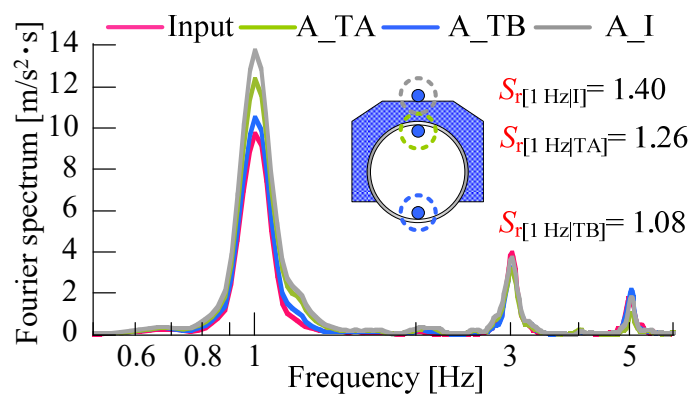

(f) Fourier spectrums (Case-3)

Figure 7. Time histories of response accelerations at tunnel and improved ground and Fourier spectrums.

\section{EXPERIMENTAL RESULTS}

\subsection{Seismic responses of tunnel and surrounding ground}

The experimental results are expressed in the prototype scale unless otherwise noted. Figures 7 (a)-(f) show the time histories of the response accelerations of the tunnels and the improved ground, and the Fourier spectrums. In the figures, the response magnification $\left(S_{\mathrm{r}}\right)$ of the Fourier spectrums at a frequency of $1 \mathrm{~Hz}$, defined by dividing the Fourier spectrum of the response acceleration by that of the input acceleration, is also shown. In terms of the Fourier spectrums and their response magnification, the response accelerations at A_TA are larger than those at A_TB in all cases, and the response magnification at A TA in Case- 2 was the maximum among all cases. It is considered that the improved ground behaves integrally with the tunnel because of the strong adhesion to the tunnel rather than to the surrounding ground. Therefore, in Case-2, where the ground around the crown of the tunnel and top section was improved, the tunnel became an unstable structure, with the weight concentrated at the upper part of the tunnel, and the inertial force acting on the tunnel increased.

Looking at the time histories of the response accelerations, A_TA responds with a delay behind A_TB in all cases. Figure 8 shows the relative response accelerations at A_TA with respect to $\mathrm{A}_{-} \mathrm{TB}$. The maximum relative response acceleration is large in the order of Case- $2>$ Case- 1 


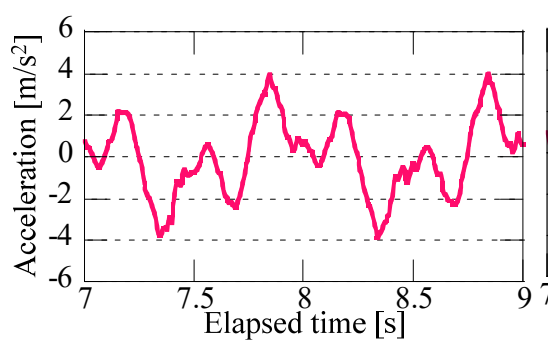

(a) Case-1

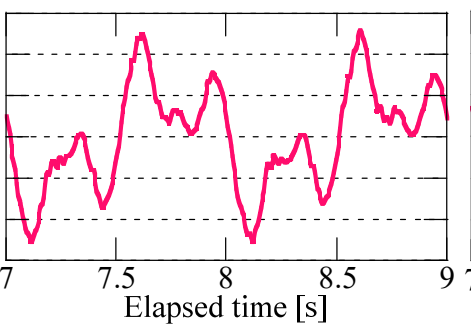

(b) Case-2

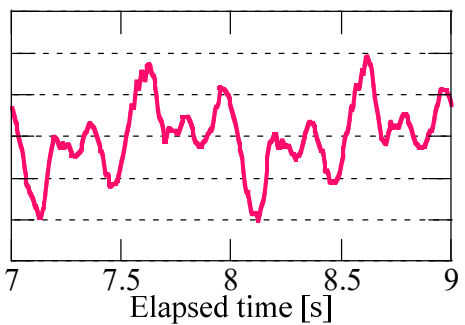

(c) Case-3

Figure 8. Time history of relative response accelerations (TB vs TA).

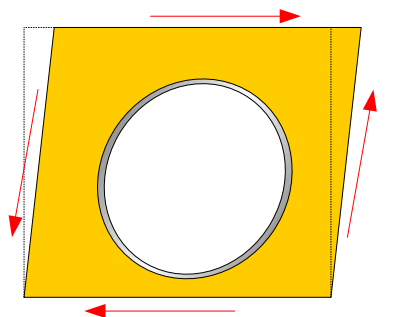

(a) Case-1

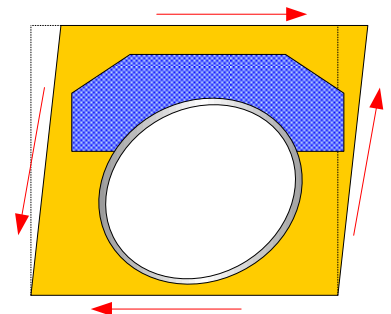

(b) Case-2

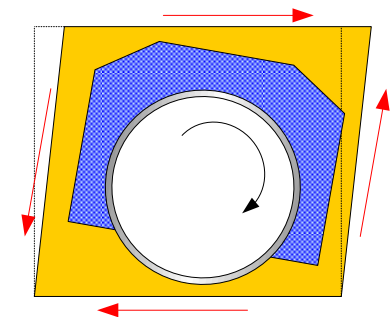

(c) Case-3

Figure 9. Expected earthquake response mode of tunnel in each case.

$>$ Case-3. It is thought that when large relative acceleration occurs, large shearing deformation occurs between the crown and the bottom of the tunnel, so the generation of a large sectional force can be expected in Case-2. Regarding the response accelerations of A_TA and A_I in Figure 7, in Case-2, both the phase and the intensity of the response accelerations are in agreement. On the other hand, in Case-3, the intensity of the response accelerations of A I is larger than that of A_TA, although these phases are almost the same. This is because, in Case-2, the response of the tunnel at the upper part is large, and the shear deformation of the tunnel excels. On the other hand, in Case-3, the shear deformation of the tunnel is suppressed by highly rigid refiners; and thus, the rotational motion is superior.

From the above considerations, the seismic behavior of a tunnel with/without ground improvement is suggested as shown in Figure 9. When the ground around the tunnel crown and top section was improved (Case-2), the response of the tunnel was amplified by the concentration of weight at the upper part of the tunnel. On the other hand, when the entire ground around the tunnel was improved (Case-3), the shear deformation of the tunnel was suppressed by increments in the whole rigidity of the surrounding ground. Thus, the rocking motion will be dominant during earthquakes.

\subsection{Section forces generated in the tunnel}

Figures 10 and 11 show the distributions of bending moments and axial forces generated in the tunnels when the right-hand side shear strain of the ground reached the maximum. The shear strain of the ground was obtained by dividing the horizontal displacement of the soil container by the wall height. A positive bending moment was defined for the case where tension was generated outside the tunnel, and the compressive axial force was defined as a positive value. The initial and residual values are also plotted in these figures.

In Case-1, the tunnel underwent oval deformation in the oblique direction by receiving shear deformation of the surrounding ground during excitation. At that time, large bending deformations occurred at the shoulder parts. At the left shoulder, positive bending moment occurred, whereas at the right shoulder, negative bending moment occurred. In addition, the axial force decreased at the left shoulder, and it increased at the right shoulder. Since this behavior is qualitatively in agreement with the behavior of a circular tunnel during an earthquake, discussed in a past research (e.g. Wang, 1993), it is confirmed that this experimental study can reproduce the seismic behavior of circular tunnels. 


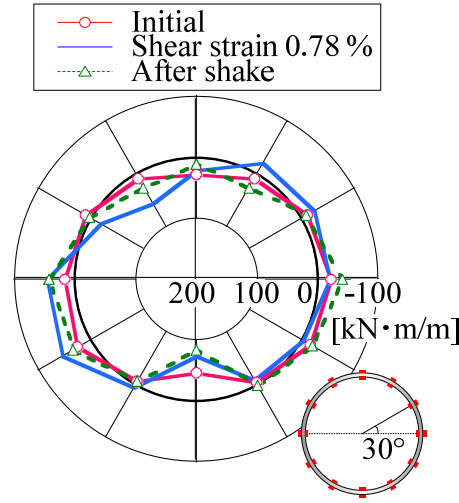

(a) Case-1

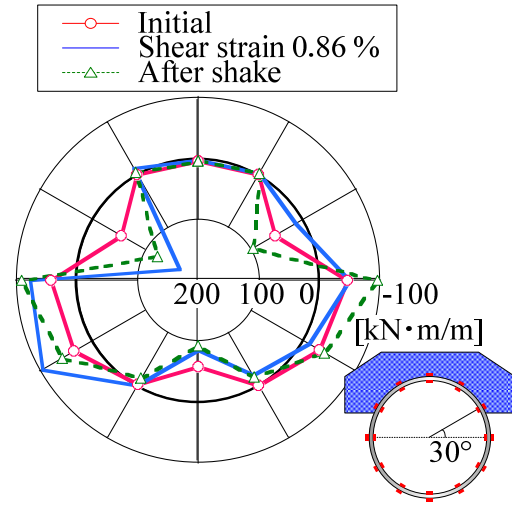

(b) Case-2

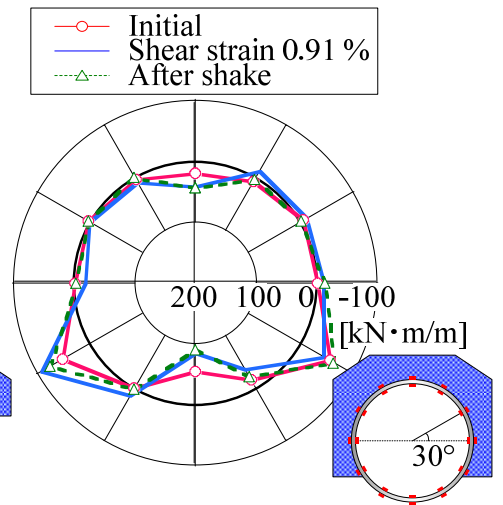

(c) Case-3

Figure 10. Distribution of bending moments generated in tunnel when right-hand side shear strain of ground reached maximum.

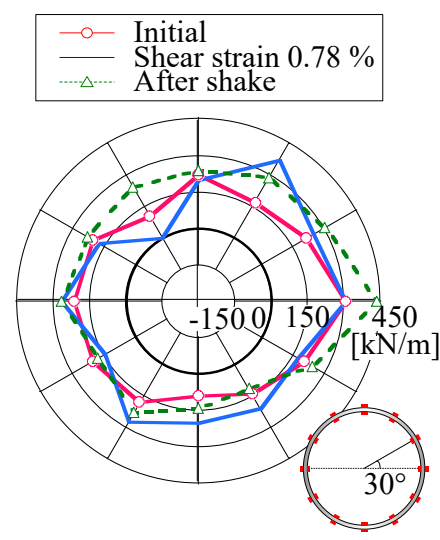

(a) Case-1

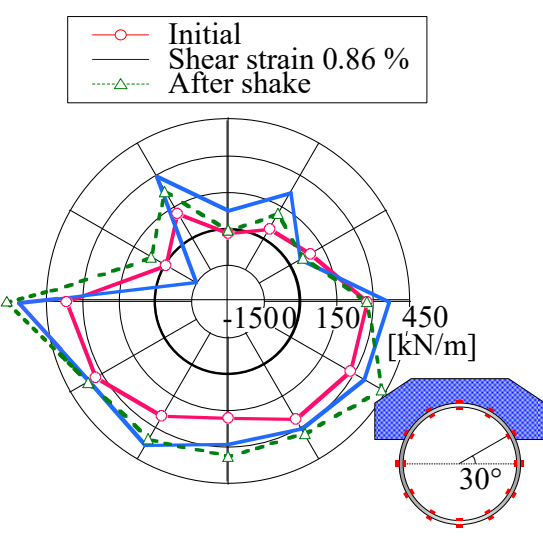

(b) Case-2

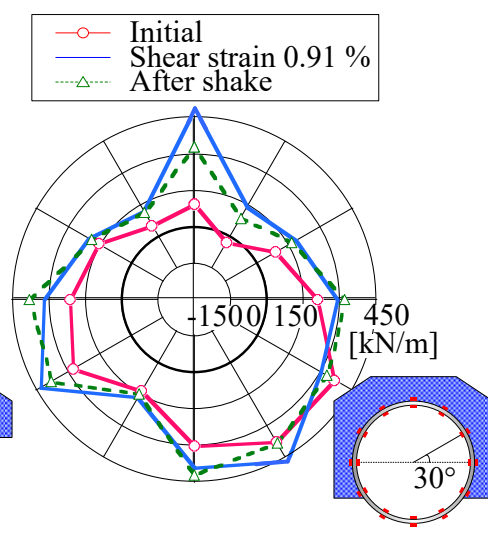

(c) Case-3

Figure 11. Distribution of axial forces generated in tunnel when right-hand side shear strain of ground reached maximum.

In Case-2, the bending moment in the region where the improved ground was placed was suppressed during vibrations. On the other hand, at the boundary between the improved ground and the surrounding ground, the sectional forces were concentrated due to the difference in rigidity, and large internal bending occurred. Moreover, the axial force decreased in these areas and tension (axial force was less than zero) occurred. In terms of the axial force generated in the lower part of the tunnel, it was smaller than in Case-1. This is considered to be due to the fact that the self-weight acting on the tunnel increased by the installation of the improved ground.

In Case-3, the bending moment in the region where the improved ground was placed was suppressed during vibrations, as it was in Case-2. The sectional forces were concentrated at the boundary of the improved ground, so that outer bending dominated the area. However, unlike Case-2, the changes in bending moment in these areas were small. In Case-3, the axial force generated at the lower part of the tunnel was large, as it was in Case-2. In addition, the increase in axial force at the crown of the tunnel was remarkable during excitation. This is because the self-weight acting on the tunnel had increased due to the installation of the improved ground. Moreover, the shearing deformation of the tunnel was suppressed by the improved ground; and thus, a large compressive force was generated at the crown of the tunnel.

From the above, it is confirmed that the bending moments generated in the tunnel were suppressed in the area where the improved ground was placed, but that large cross-sectional forces were generated at the boundary between the improved ground and the unimproved ground. On the other hand, it was revealed that when the improved ground was placed, the self-weight acting on the tunnel increased and the compressive force generated in the lower part of the tunnel increased. 


\section{CONCLUSIONS}

In this study, dynamic centrifugal model experiments under a gravitational acceleration of $50 \mathrm{G}$ were conducted to clarify the dynamic behavior of shallow tunnels with pre-ground improvement. Two ground improvement patterns were investigated: (1) the ground around all the crosssections of the tunnel was improved and (2) the ground around the crown of the tunnel and top section was improved. The results from these two patterns were compared with a case without ground improvement. The findings obtained from this research are as follows.

1) When the ground around the tunnel crown and top section was improved (Case-2), the response of the tunnel was amplified by the concentration of weight at the upper part of the tunnel. As a result, the different phases between the responses at the bottom and the top of the tunnel increased, causing large shear deformation of the tunnel at the boundary between the improved ground and the unimproved ground.

2) When the entire ground around the tunnel was improved (Case-3), the shear deformation of the tunnel could be suppressed by increments in the whole rigidity of the surrounding ground. Accordingly, it was found that the rocking motion of the tunnel would be dominant during earthquakes.

3) Although the improved ground preserved the shear deformation of the tunnel, large crosssectional forces were generated at the boundary between the improved ground and the unimproved ground due to the difference in rigidity. Therefore, it can be said that the earthquake resistance of a shallow overburden tunnel can be improved when the entire ground around the tunnel is improved. On the other hand, when the ground around the tunnel crown and top section is improved, there is a possibility of damage to the tunnel at the boundary between the improved ground and the unimproved ground.

\section{REFERENCES}

Cui, Y., Kishida, K. \& Kimura, M. 2018. Numerical study on the effect of pre-ground improvement method on control of ground subsidence occurring in shallow overburden NATM tunnels. Proc. of the 7th China-Japan Geotechnical Symposium, 31-37, Sanya, 16-18 March 2018.

Hashash, Y. M. A., Hook, J. J., Schmidt, B. \& Yao, J. I. C. 2001. Seismic design and analysis of underground structure. Tunnelling and Underground Space Technology, 16, 247-293.

Jiang, Y.J., Wang, C.X. \& Zhao, X.D. 2010. Damage assessment of tunnels caused by the 2004 Mid Niigata Prefecture Earthquake using Hayashi's quantification theory type II, Natural Hazards, 53(3), 425-441.

Kishida, K., Cui, Y., Nonomura, M., Iura, T. \& Kimura, M. 2013. Discussion on the mechanism of ground improvement method at the excavation of shallow overburden tunnel in difficult ground, Underground Space, 1(2), 94-107.

Konagai, K., Takatsu, S., Kanai, T., Fujita, T., Ikeda, T. \& Johansson, J. 2009. Kizawa tunnel cracked on 23 October 2004 Mid-Niigata earthquake: An example of earthquake-induced damage to tunnels in active-folding zones. Soil Dynamics and Earthquake Engineering, 29 (2), 394-403.

Nonomura, M., Iura, T., Cui, Y., Kishida, K. \& Kimura, M. 2013. Discussion on mechanical evaluation of ground pre-improvement method under shallow overburden and unconsolidated ground. Japanese Geotechnical Journal, 8(2), 165-177. (in Japanese)

Shen, Y.S., Gao, B., Yang, X.M. \& Tao, S.J. 2014. Seismic damage mechanism and dynamic deformation characteristic analysis of mountain tunnel after Wenchuan earthquake. Engineering Geology, 180, 8598.

Wang, J. N. 1993. Seismic design of tunnels: A state-of-the-art approach. Monograph 7, Parsons Brinckerhoff Quade \& Douglas, New York.

Wang, Z. Z., Gao, B., Jiang, Y.J. \& Yuan, S. 2009. Investigation and assessment on mountain tunnels and geotechnical damage after the Wenchuan earthquake. Science in China Series E: Technological Sciences, 52 (2), 546-558.

Yashiro, K., Kojima, Y. \& Shimizu, M. 2007. Historical earthquake damage to tunnels in Japan and case studies of railway tunnels in the 2004 Niigataken-Chuetsu earthquake. Quarterly Report of RTRI, 48(3), 136-141.

Zhang, X. P., Jiang, Y. J. \& Sugimoto, S. 2018. Seismic damage assessment of mountain tunnel: a case study on the Tawarayama tunnel due to the 2016 Kumamoto Earthquake. Tunnelling and Underground Space Technology, 71, 138-148. 